\title{
Quale futuro per il patrimonio fortificato: problemi di conservazione e valorizzazione
}

What future for fortified heritage: problems of conservation and enhancement

\section{Rossella de Cadilhac}

Politecnico di Bari, Bari, Italy, decadiross@gmail.com / rossella.decadilhac@poliba.it

\begin{abstract}
What are the risk factors that undermine the conservation of fortified architecture? What are the future prospects of a heritage that is not always adequately protected and valued? These are the questions posed by castles, towers, fortresses that seem to have lost the meaning of their existence, for having failed in their original function. Certainly the abandonment involves an inevitable lack of maintenance with the inevitable decay due to natural causes with slow action and prolonged over time. But even bad restorations conducted without the necessary historical-critical awareness and the necessary technical skills can irreversibly cancel documents of art and history, as well as "material testimonies of civilization value". It is a heritage of multiple criticalities that offers an extraordinary opportunity for contemporary design aiming at conservation. Innumerable problems are posed by fortified architectures, first and foremost the accessibility, but also their management, which cannot but take into account the very close typology-function relationship and where, however, the old utilization is no longer possible. Unquestionably the first step is represented by the knowledge of these singular architectures, in the becoming of historical processes, which is an unavoidable moment on which the choices aiming at conservation and valorisation depend. Emblematic from this point of view are some case studies investigated in Puglia which pose the problem of maximum conservative attention and desirable enhancement, pursued with the promotion of knowledge and the assurance of the best conditions of use, provided that the new function is respectful of the identity of the object of study, of its use vocations and attentive to the needs and expectations of the community.
\end{abstract}

Keywords: Fortified architecture, abandonment, conservation, enhancement.

\section{Introduzione}

Il frazionamento della proprietà -pubblica o privata che sia-, una scarsa sensibilità derivata da una mancanza di educazione al sentimento del bene di interesse storico e artistico, una generale impreparazione delle professionalità e una diffusa inadeguatezza delle maestranze, oltre ad interessi speculativi, sono fra i fattori di rischio che attualmente minano la conservazione del patrimonio fortificato, non sempre adeguatamente tu-

telato e valorizzato. Va segnalato inoltre che la conoscenza del tema delle fortificazioni, come di tutto il patrimonio architettonico, fa i conti con un approccio al rilievo stratigrafico degli elevati ancora inmaturo, come giustamente confermato dagli studi, tra gli altri, di Brogiuolo e Cagnana. Un'archeologia dell'architettura di larghi orizzonti a livello interdisciplinare garantirebbe al contrario pratiche di analisi assolutamente indi- 
spensabili alla comprensione di palinsesti di grande ricchezza come in genere sono i castelli, tanto più antichi, spesso, quanto più oggetto di "aggiornamenti" che ne hanno aumentato la sapienza costruttiva e la cultura.

Tutte queste circostanze sono alla base di situazioni di abbandono, di sottoutilizzo oppure, al contrario, di contraffazione che, portate alle estreme conseguenze, mettono a dura prova la sopravvivenza stessa di quel patrimonio. È evidente, allora, che l'educazione all'arte e al sentimento del bene d'interesse collettivo e la conoscenza debbano essere riconquistati come momenti essenziali, da un lato per informaresensibilizzare le coscienze, dunque "promuovere lo sviluppo della cultura"1, dall'altro lato per formare l'architetto chiamato ad intervenire con consapevolezza storico-critica e competenza tecnica su preesistenze da salvaguardare e valorizzare.

Per questa ragione il quadro delle conoscenze diventa il momento propedeutico ad ogni azione conservativa. Interessarsi degli aspetti materiali e costruttivi che sostanziano le opere fortificate, arrivando a comprendere la loro essenza più intima, significa creare le premesse per mettere a punto operazioni tecniche attente e scrupolose al fine di preservarle dai processi di alterazione e deterioramento. Come pure cogliere e comprendere gli aspetti estetico-percettivi legati all'immagine delle architetture munite, vuol dire riconoscerne il valore figurativo che come tale va tutelato e valorizzato.

Infine, acquisire dati sugli aspetti funzionali, che implica il dover affrontare questioni legate a problemi di riuso e accessibilità, prelude alla possibilità di rendere vitali queste opere attraverso un progetto di rinnovata fruizione nell'ambito di una più ampia strategia di sviluppo a scala territoriale.

\section{La parabola dei castelli}

Le architetture fortificate sono fra quelle espressioni del fare umano che nel tempo hanno perso gradualmente le ragioni della loro esistenza per il fatto di essersi allontanate dalle originarie motivazioni, strettamente utilitarie, che hanno fortemente condizionando il loro destino.
La variabilità del giudizio di valore ha certamente risentito della parábola funzionale che ha accompagnato i manufatti difensivi, dalle prime formulazioni, al loro potenziamento, fino al definitivo abbandono per la sopraggiunta inutilità (Mariotti, 2017, pp. 15-17). Un sostanziale disinteresse alla loro conservazione materiale si registra nel corso del XVI secolo, dopo l'affermarsi del cosiddetto fronte bastionato, con il progressivo esaurimento della funzione di macchina bellica e la perdita del proprio ruolo strategicomilitare, che ha portato in alcuni casi all'abbandono, in altri ad intenzionali demolizioni, in altri ancora alla trasformazione in dimore signorili, istituti carcerari o caserme.

Una rinnovata attenzione si manifesta fra il XVIII e il XIX secolo nel contesto del gothic revival con il ridestarsi dell'interesse nei confronti delle opere fortificate, quando i castelli medievali diventano, da un lato luoghi di ambientazione di romanzi fantastici che inaugurano il filone letterario delle gothic stories (Walpole, 1764), dall'altro oggetto dei primi restauri oscillanti fra principi stilistici secondo la tradizione d'oltralpe e criteri filologici di matrice boitiana. È nel contesto di un'Italia appena unita, ma ancora priva di un'identità nazionale, che matura la convinzione di affidare ad un giudizio di valore altamente selettivo la scelta di emergenze architettoniche -è il caso dei restauri del castello sforzesco di Milano o del castello di Fenis- che fossero rappresentative e, in quanto tali, meritevoli di conservazione.

Bisogna attendere gli anni Sessanta del XX secolo per vedere ampliato l'orizzonte della tutela, con un'importante ricaduta sul destino delle architetture fortificate ${ }^{2}$, per l'estensione del concetto di monumento storico -dalle emergenze architettoniche alle opere modeste, "dalla creazione isolata all'ambiente urbano e paesistico che costituisca testimonianza di una civiltà particolare, di un'evoluzione significativa, di un avvenimento storico"-, e l'affermarsi della tutela attiva cioè della cosiddetta conservazione 'in vita' attraverso la scelta di un uso/riuso corretto, finalizzato alla fruizione pubblica nel momento presente pur sempre nel rispetto della materia antica, dell'unità figurativa e del rapporto con il contesto ambientale di appartenenza. 


\section{Istanze conservative e prospettive di valo- rizzazione}

Arrivare a riconoscere alle architetture fortificate valori (storico-artistici, ambientali-paesaggistici, socio-economici) (Mariotti, 2017, pp. 51-52) che trascendono le ragioni utilitarie per le quali erano state concepite e realizzate, significa aprire nuove prospettive in cui il passato può saldarsi al futuro attraverso la "tutela" e la "valorizzazione". La prima, attribuendo un valore storico, artistico, oltre che psicologico, al patrimonio fortificato inscindibilmente legato al suo ambiente, si pone l'obiettivo di proteggerlo per consegnarlo inalterato alle generazioni future. È così che l'inutilità delle strutture difensive come macchine militari e la loro sostanziale estraneità rispetto all'attuale momento storico vengono superate dai messaggi immateriali che esse veicolano, gli unici che soddisfano le esigenze spirituali dell'uomo, cui ricondurre le più profonde ragioni della conservazione. La seconda, mai disgiunta dalla prima, è orientata a svilupparne le potenzialità, favorendo la diffusione della conoscenza, l'estensione del parterre dei destinatari e promuovendo la fruizione pubblica attraverso la ricerca di una funzione rispettosa della vocazione collettiva, integrata nella vita sociale e compatibile con gli antichi aspetti distributivi. $\mathrm{Si}$ tratta di restituire una rinnovata vitalità, reimmettendo le strutture difensive nel circuito della fruizione attuale attraverso una reinterpretazione nel tempo presente, con l'obiettivo di potenziare le peculiarità storiche e artistiche che fanno di quelle architetture un unicum irripetibile. Ma l'azione di tutela si rivelerebbe inefficace se si ignorasse l'incidenza del valore economico, che permette di associare al valore culturale, beninteso riconosciuto sempre come prevalente, un beneficio sociale e una opportunità economica.

Malgrado l'impossibilità di assicurare una continuità d'uso alle architetture fortificate crei un vulnus che le eccettua dalla generalità delle opere del passato, ponendo con ciò delicate questioni di riuso, si è concordi nel ritenere che non si può parlare di un restauro ad hoc per i manufatti difensivi, continuando a sussistere la fondatezza dell'unità metodologica del fare restauro (Baldini, 1978-1981; Brandi, 1977; Carbonara, 1976, 2013, pp. 93-102, 2016, pp. 83-88). Ciò non to- glie che si debbano riconoscere delle specificità ai manufatti difensivi cui rivolgere la massima attenzione, come ad esempio gli apparati decorativi che -per l'assoluta coerenza fra forma e struttura- sono la manifesta espressione della propria funzione, o gli aspetti distributivi pienamente conformi allo strettissimo rapporto tipologia-funzione (Varagnoli, 2016, pp. 135-141). Allora, da un lato si pone il problema della conservazione della materia antica, dei segni delle stratificazioni storiche, delle peculiarità costruttive e decorative che vanno comprese, preservate e valorizzate, dall'altro la questione della ricerca di una funzione appropriata assicurando le necessarie condizioni di accessibilità e vivibilità, all'interno di una più ampia strategia di promozione e sviluppo dei valori storico-culturali che le architetture fortificate custodiscono ed esprimono.

\section{Criticità e potenzialità nella lettura di casi emblematici}

Emblematici sono alcuni casi di studio indagati in Puglia i quali pongono il problema della massima attenzione conservativa e dell'auspicabile valorizzazione, perseguibili con la promozione della conoscenza e l'assicurazione delle migliori condizioni di uso, purché la nuova funzione sia rispettosa dell'identità dell'oggetto di studio, delle sue vocazioni ed attenta ai bisogni e alle aspettative della collettività.

Condizioni di incertezza legate al sottoutilizzo connotano il castello di Massafra (TA) (Cardone, et al., 2013; Cadilhac, 2017, pp. 89-96) (Figg. 1 e 2), ora di proprietà comunale e solo parzialmente fruito per aver accolto in un suo braccio la biblioteca comunale. Nella sua attuale configurazione il castello è il risultato di trasformazioni successive, spesso dettate da esigenze di carattere funzionale che hanno variato il nucleo più antico reinterpretandolo continuamente, fino al raggiungimento di un'immagine ora consolidata e sedimentata nella memoria collettiva, nonostante incauti interventi abbiano messo a dura prova lo stato di conservazione. È un ricco palinsesto architettonico quello che la storia ci restituisce, che ora soffre la condizione di parziale abbandono e che mostra i segni del tempo trascorso, portatori di messaggi unici e irripetibili, il cui progetto di restauro deve conservare per perpetuarne il riconoscimento. 


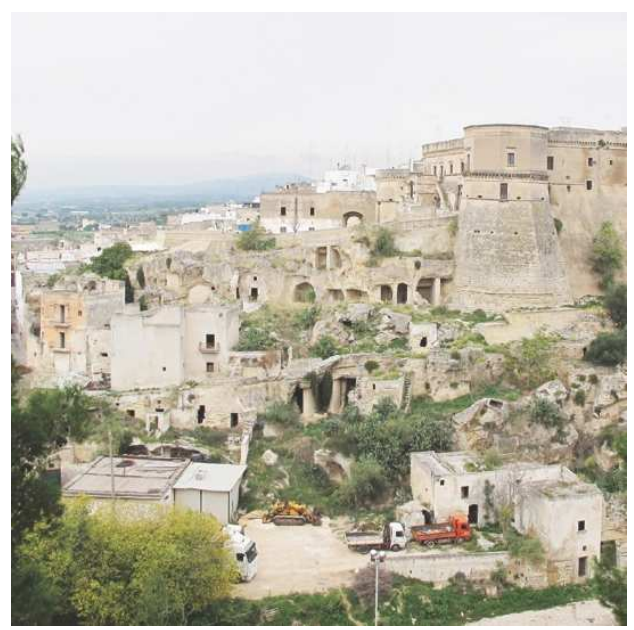

Fig. 1. Massafra (TA), il Castello e il contesto paesaggistico (Cardone, et al., 2013).

Ma l'oggetto di studio offre anche l'occasione per riflettere sul delicato rapporto conservazione / innovazione, incentrato sui problemi d'integrazione, sostituzione, ricostruzione, dalla scala architettonica a quella urbana e paesistica, tutti temi che nel caso specifico, possono essere risolti in nome della continuità con il passato, nel rispetto del principio del minimo intervento, della compatibilità, della riconoscibilità, senza rinunciare con questo alle capacità espressive del linguaggio contemporaneo. Lo stato di degrado in cui versa gran parte del castello, il preoccupante quadro fessurativo, le manomissioni anche recenti cui è stato sottoposto, suggeriscono lo spunto per un progetto complessivo, unitario e coerente, che, nel riammettere il complesso architettonico nel circuito della fruizione, in linea con le strategie comunali orientate alla conservazione del proprio patrimonio architettonico, ne rispetti il carattere identitario valorizzando le proprie vocazioni funzionali. La consolidata tradizione legata alla cinematografica comprovata dall'ampio consenso raccolto in seguito a festival e rassegne dedicati al cinema, ben si coniugherebbe con l'esigenza di creare centri specializzati nella post produzione cinematografica allo scopo di soddisfare la crescente domanda che deriva dall'afflusso di troupe cinematografiche nella regione. Potrebbe essere questa l'occasione per attribuire al castello il ruolo di custode e promotore della cultura e della tradizione locale, capace di valorizzare la città ed il suo territorio, entrambi inseriti all'interno dell'avviato circuito cinematografico regionale.

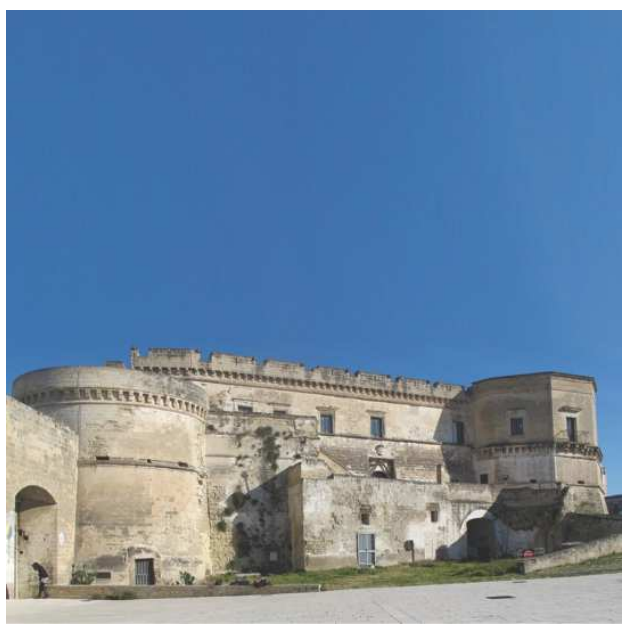

Fig. 2. Massafra (TA), il prospetto sud del Castello (Cardone, et al., 2013).

Fattori di rischio per il castello di Otranto (LE) (Baldassarre, et al., 2018) (Figg. 3 e 4) sono l'insufficiente valorizzazione nonostante il buono stato di conservazione generale, ma ancor più la parziale compromissione del rapporto spaziale, con conseguenti problemi di accessibilità, fra il complesso architettonico, il fossato, la cinta urbana e il centro antico; il che mette in evidenza alcuni nodi problematici a cui si dovrebbe poter dare una risposta in termini progettuali. Un tema cruciale dal punto di vista della raggiungibilità è rappresentato dalla Porta Nova Ferrata, più nota come Porta a Mare, aperta in breccia sul tratto del circuito fortificato che collega il castello alla Torre Matta in prossimità del porto e che segna il punto di accesso alla città da Est. Più problematico è il lato interno della porta urbana dove convivono il fornice del varco e i resti della Chiesa settecentesca dedicata all'Immacolata crollata negli anni Settanta del XX secolo, di cui sono ancora riconoscibili le tracce della parete absidale e delle due cappelle adiacenti scavate nello spessore della cortina muraria. Un'ipotesi percorribile sarebbe quella di riscattare il fossato dalle condizioni di isolamento, puntando su un tragitto che dalla Porta Alfonsina scende alla quota più bassa lungo il circuito fortificato dell' antemurale, raggiunge il castello e prosegue verso il porto.

Il manufatto difensivo che costituirebbe il fulcro del percorso di visita offrirebbe l'occasione per riflettere sull'opportunità della sua valorizzazione attraverso una parziale riconversione d'uso, che sia rispettosa dell'articolazione spaziale e 
dell'individualità espressiva del complesso monumentale. In sintonia con le risorse, i bisogni e le aspirazioni della comunità, il castello può diventare un polo culturale, capace di attrarre abitanti del luogo e turisti guidati lungo un itinerario alla scoperta delle fasi costruttive di questo palinsesto dinamico, condizionate dall'evoluzione dell'arte poliorcetica e delle tecniche ossidionali; della storia evolutiva della città di Otranto e del suo territorio; della cultura del luogo, attraverso racconti letterari interattivi che, non escludendo l'aspetto ludico, potrebbero coinvolgere le varie forme dell'espressione artistica.

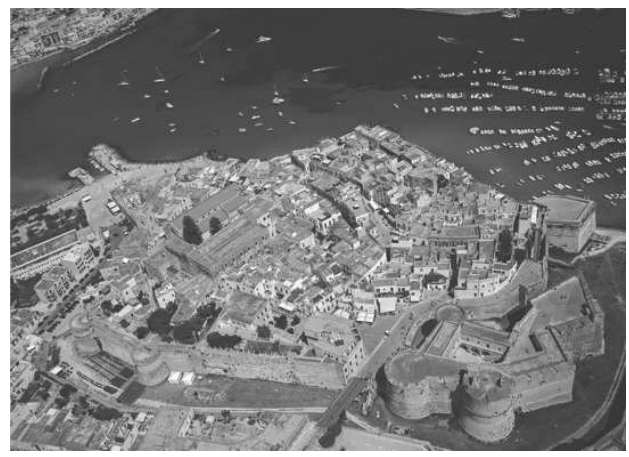

Fig. 3. Otranto (LE), il Castello e il centro antico (Baldassarre, et al., 2018).

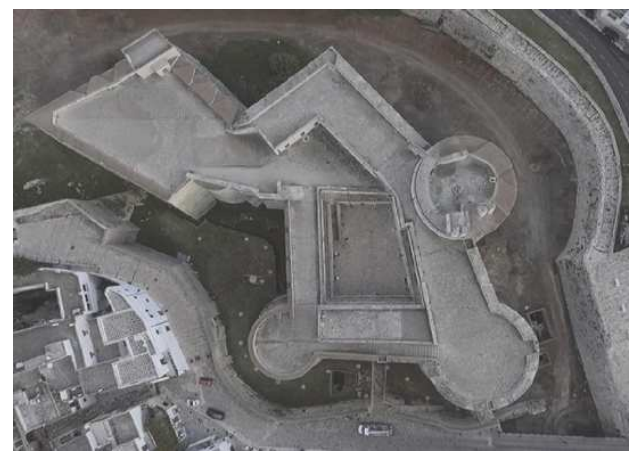

Fig. 4. Otranto (LE), configurazione planimetrica del Castello e del fossato (Baldassarre, et al., 2018).

Criticità, in parte riconducibili ad azioni antropiche, si registrano nel castello di Monte Sant'Angelo (FG) (Bruni, et al., 2019) (Figg. 5 e $6)$, che versa in uno stato di semiabbandono e sottoutilizzo, sebbene di recente l'amministrazione comunale abbia affidato la gestione all'Ente Parco Nazionale del Gargano con l'obiettivo di restituire a nuova vita il complesso fortificato. Elemento strategico di un articolato sistema di difesa territoriale fatto di torri di avvistamento, torri costiere, castelli e masserie fortificate, il complesso monumentale, persa la sua funzione originaria, sembra aver smarrito la ragione della propria esistenza. Innumerevoli sono le trasformazioni che, in un processo di lunga durata, hanno mutato il dato originario modificandolo ininterrottamente, con aggiunte, sottrazioni, manipolazioni, spesso legate a cambi di destinazione d'uso, crolli imputabili a periodi di abbandono, restauri non sempre congrui che hanno compromesso sia la statica che l'estetica dell'organismo architettonico. Nell'ambito di un più ampio progetto di valorizzazione del paesaggio, il castello potrebbe costituire un rinnovato elemento di riferimento e richiamo territoriale puntando sulla necessità di instaurare un rapporto di reciproca necessità fra monumento e territorio, ma anche fra monumento e collettività se esso venisse promosso come centro propulsore di conoscenza del patrimonio naturalistico e della tradizione culturale del luogo, ma anche "generatore di partecipazione sociale". Per raggiungere questa finalità, si ritiene debba essere restituita dignità architettonica al complesso fortificato compromesso da interventi che nel tempo ne hanno pregiudicato il valore espressivo. Ad esempio, potrebbe essere restituita la continuità fisica e percettiva del cammino di ronda interrotto in più tratti, vista la incontrovertibilità delle tracce materiali, attraverso una reintegrazione che affida la sua cifra stilistica ad un sistema riconoscibile, compatibile e potenzialmente reversibile. Mentre potrebbe essere resa nuovamente fruibile la Torre Quadrata Est inspiegabilmente svuotata e ridotta ad un banale involucro murario attraverso una reinterpretazione dell'articolazione spaziale interna -diversa da quella originaria-, in quanto non più riproponibile per insufficienza di indizi, ma in continuità con l'esistente.

Il progetto non dovrà trascurare il problema della fruizione proponendo un'esplorazione dei luoghi attraverso un "nastro" continuo, riconoscibile attraverso accorgimenti fisici e visivi, il quale potrebbe snodarsi dall'ingresso, ai cortili, fino agli ambienti interni, guidando l'ipotetico visitatore alla scoperta e comprensione dei segni delle stratificazioni storiche. Restituita l'unità architettonica al complesso fortificato, si dovrà affrontare la re-immissione nel circuito della fruizione con la conversione del castello da 
avamposto strategico di difesa del Gargano a custode del patrimonio naturalistico e promotore alla scala territoriale della specificità culturale del luogo.

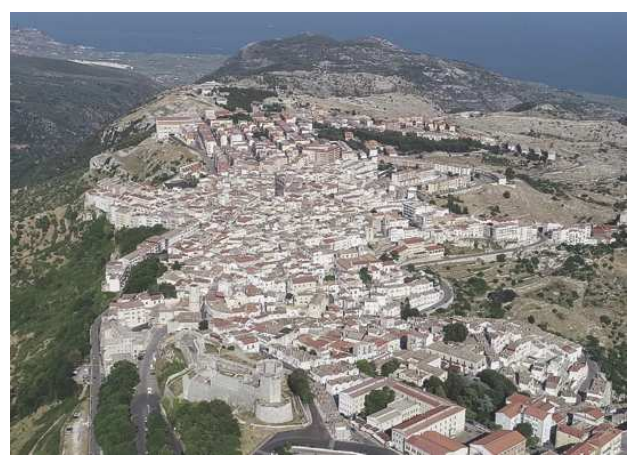

Fig. 5. Monte Sant'Angelo (FG), il Castello e il contesto paesaggistico (Bruni, et al., 2019).

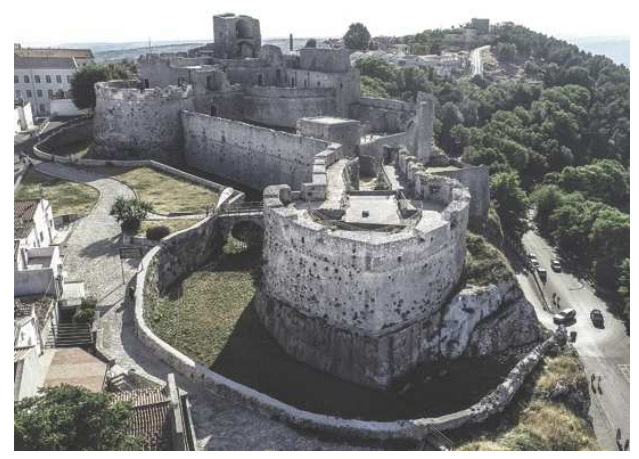

Fig. 6. Monte Sant'Angelo (FG), l'articolato sistema difensivo del Castello (Bruni, et al., 2019).

\section{Conclusioni}

Ogni azione finalizzata alla tutela e valorizzazione delle architetture fortificate dovrà fare riferimento ad un'unica base concettuale in quanto esse non possono eccettuarsi dal più generale patrimonio architettonico. Tutela e valorizzazione non sono separabili, l'una non può sussistere senza l'altra. Se la tutela dovrà proteggere il patrimonio per consegnarlo al futuro impedendo il degrado della materia, della struttura fisica e del contenuto culturale, la seconda dovrà "conferire valore" per far esprimere al patrimonio le proprie potenzialità e favorire la fruizione che a sua volta sarà facilitata se verrà migliorato il suo stato di conservazione generale.

\section{Note \\ ${ }^{1}$ Decreto Legislativo 22 gennaio 2004, n. 42, no- to come "Codice dei beni culturali e del paesag- gio". \\ ${ }^{2} \mathrm{Nel} 1964$ viene sottoscritta la Carta internazio- nale del restauro (o carta di Venezia) e fondato l'IIC su iniziativa di Piero Gazzola.}

\section{Bibliography}

Baldassarre, V; et al. (2018). Sistemi fortificati in Terra d'Otranto. Studi analitici e percorsi interpretativi, Tesi di laurea in Restauro Architettonico, Politecnico di Bari, Edizioni Grafiche Favia, Modugno.

Baldini, U. (1978-1981). Teoria del restauro e unità di metodologia, Nardini Editore, Firenze, vol. I-II.

Beltramo, S. (2009). Stratigrafia dell'architettura e ricerca storica, Carocci, Roma.

Boato, A. (2004). Indagini di archeologia dell'architettura su un edificio pluristratificato, San Giorgio, Genova.

Brandi, C. (1977). Teoria del restauro, Piccola Biblioteca Einaudi, Torino.

Brogiuolo., P.; Cagnana, A. (2017). Archeologia dell'architettura: metodi ed interpretazioni, All'insegna del Giglio, Firenze.

Bruni, G.; et al. (2019). Nuovi orizzonti di conservazione e valorizzazione del patrimonio fortificato. Il caso del castello di Monte Sant'Angelo in Capitanata, Tesi di laurea in Restauro Architettonico, Politecnico di Bari, Copy@net Centro stampa digitale, Bari.

Cadilhac, R. de. (2017). "Architetture fortificate in Terra d'Otranto. Il ruolo del restauro fra istanze conservative e fruizione cosapevole", in Architettura fortificata. Rilievo e restauro, Aesei editore, Martina Franca. 
Carbonara, G. (1976). La reintegrazione dell'immagine. Problemi di restauro dei monumenti, Bulzoni, Roma.

Carbonara, G. (2013). "Riflessioni sull'unità di metodo nel restauro", in Architettura d'oggi e restauro. Un confronto antico-nuovo, Utet, Torino.

Carbonara, G. (2016). "Architetture fortificate: restauro e valorizzazione", in Architettura fortificata. Rilievo e restauro, Aesei editore, Martina Franca.

Cardone, F.; et al. (2013). Progetto di restauro del castello di Massafra (TA), Tesi di laurea in Restauro Architettonico, Politecnico di Bari, Copy@ net Centro stampa digitale, Bari.

Cavada, E.; Genitilini, G. (2007). Il restauro dei castelli: analisi e interventi sulle architetture fortificate: conoscere per restaurare. Atti dei seminari in archeologia dell'architettura, Trento 2002-2004, Centro duplicazione della provincia autonoma di Trento, Trento.

Cecchini, L. (2009). Natura e archeologia al fondamento dell'architettura, Alinea, Firenze.

Esposito, D. (1997). “Carte, documenti e leggi”, in Trattato di Restauro Architettonico, Utet, Torino, vol. IV.

Mariotti, C. (2017). Il restauro dei castelli in Italia: 1964-2014, Tesi di dottorato in Architettura, Università di Bologna, Bologna.

Pittaluga, D. (2009). Questioni di archeologia dell'architettura e restauro, ECIG, Genova.

Varagnoli, C. (2016). "L'avvenire dei castelli: riflessioni ed esperienze”, in Architettura fortificata. Rilievo e restauro, Aesei editore, Martina Franca.

Walpole, H. (1794). The Castle of Otranot. A Gotic story, Himburg, Berlin. 
